\title{
La Chronique de Dalimil et les débuts de l'historiographie nationale tchèque en langue vulgaire
}

\section{Éloïse Adde}

\section{(2) OpenEdition \\ Journals}

Édition électronique

URL : http://journals.openedition.org/ifha/173

DOI : $10.4000 /$ ifha. 173

ISSN : 2198-8943

\section{Éditeur}

IFRA - Institut franco-allemand (sciences historiques et sociales)

\section{Édition imprimée}

Date de publication : 6 février 2011

Pagination : 119-125

ISSN : 2190-0078

\section{Référence électronique}

Éloïse Adde, « La Chronique de Dalimil et les débuts de l'historiographie nationale tchèque en langue vulgaire », Revue de l'IFHA [En ligne], 3 | 2011, mis en ligne le 10 février 2012, consulté le 20 avril 2019. URL : http://journals.openedition.org/ifha/173 ; DOI : 10.4000/ifha.173

Ce document a été généré automatiquement le 20 avril 2019.

(CIFHA 


\title{
La Chronique de Dalimil et les débuts de l'historiographie nationale tchèque en langue vulgaire
}

\author{
Éloïse Adde
}

\section{NOTE DE L'ÉDITEUR}

Éloïse Adde a soutenu sa thèse de doctorat sous la codirection de Jean-Philippe Genet et Xavier Galmiche le 25 juin 2011, à l'université Paris I. Elle a été bénéficiaire d'une aide à la mobilité à l'IFHA de septembre 2009 à août 2010. Depuis septembre 2011, elle est chercheur au Centre français de recherche en sciences sociales (Cefres, Prague).

1 Cette analyse s'appuie sur la Chronique de Dalimil, première chronique rédigée en tchèque au début du XIVe siècle. Nous avons pris comme point de départ la problématique posée par la naissance d'une littérature en langue vulgaire dans l'Europe chrétienne, pour réfléchir sur les formes prises par cette littérature nouvelle et le public qu'elle vise; et, en amont, pour déterminer quels facteurs ont été décisifs et quelles réponses les auteurs entendaient apporter à travers une telle initiative.

La Chronique de Dalimil est un document majeur: il s'agit du premier texte historiographique écrit en langue vulgaire (vers 1309-1311); en outre, il correspond au deuxième texte littéraire écrit en tchèque, suivant de quelques années l'Alexandreis, adaptation en tchèque du Roman d'Alexandre de Gauthier de Châtillon (vers 1250-1300).

Son auteur est pourtant inconnu. Le nom de Dalimil est entré par hasard dans l'histoire. Longtemps, le texte qu'il nous livra s'appela simplement Kronika (Chronique), voire Kronika ãeská (Chronique tchèque). C'est le manuscrit de Fürstenberg (1400-1425) qui fut à l'origine d'un amalgame tenace, en faisant figurer comme titre de la chronique le nom de Kronika boleslavská, c'est-à-dire Chronique de Boleslav. Parmi les sources qu'il avait utilisées pour rédiger sa célèbre chronique, Václav Hájek de Liboãany citait en 1541 un certain Dalimil de Mezefiice, chanoine de Boleslav, et Tomáš Pešina de âechorod eut tôt fait de ne faire 
qu'un de ce texte et de ce présumé auteur plus d'un siècle plus tard (1673-1677). Même si Josef Dobrovský (1753-1829) et František Palacký (1798-1876) prouvèrent qu'il s'agissait d'une erreur, le nom passa à la postérité, propulsé sur le devant de la scène par le succès de la chronique qui lui était associée, particulièrement à l'époque du Renouveau national tchèque et des efforts réalisés par les intellectuels tchèques pour renouer avec leur identité propre durant le XIXe siècle. Aujourd'hui, même si l'erreur sur le nom ne fait plus l'ombre d'un doute, ce dernier reste utilisé par souci de commodité. Nous-même préférons parler de la Chronique de Dalimil.

4 Ce texte rimé contient 103 chapitres qui relatent l'histoire de la Bohême, de ses origines mythiques jusqu'à l'époque contemporaine de sa rédaction. Si nous excluons la préface, qui répond aux canons de l'époque en justifiant les choix et les intention de l'auteur, nous pouvons distinguer trois parties: la première concerne l'histoire légendaire, avec le mythe ethnogénétique et l'arrivée des Tchèques en Bohême, ainsi que le cycle de Libuše qui rend compte de l'apparition de «l'État » tchèque (chap. 1-24); la deuxième consiste en l'histoire puisée dans les sources (chap. 25-77), tandis que la troisième correspond aux événement auxquels l'auteur a assisté en personne (chap. 78-103). Nous pouvons superposer sur les deux dernières parties un autre découpage, avec une première section qui narre l'ascension de la Bohême (chap. 25-75) et une deuxième qui relate son déclin, à partir du règne de Przemysl Ottokar Ier (chap. 75-103).

5 Malgré un recours aux épisodes bibliques et aux motifs mythologiques, Dalimil propose à ses lecteurs une vision de l'histoire tout à fait novatrice par rapport à la tradition historiographique tchèque fondée par Cosmas (1125). Cette vision rompt en effet avec l'histoire contemplative comme description de l'accomplissement des plans divins, pour livrer un message engagé politiquement, tourné vers l'avenir et revisitant le passé à l'aune des événements du présent. Dans un contexte où la menace allemande est bien réelle, surtout depuis l'extinction de la dynastie des Przemyslides en 1306, l'auteur entend mobiliser la "nation " tchèque contre l'ennemi, en rappelant que la présence allemande en Bohême a toujours été synonyme de malheur pour le pays. Le texte est donc empreint d'un nationalisme et d'une agressivité envers les Allemands extrêmement acérés et tout à fait originaux pour l'époque.

6 Nous avons divisé ce travail en deux volumes. Le second est dédié à la traduction sur laquelle nous reviendrons en fin de présentation; le premier contient la thèse proprement dite et se compose de trois grandes parties intitulées : 1 . "Sources, textes et contextes»; 2. "Identité collective, nationalisme et xénophobie»; 3. «Société et le programme politique ».

7 Nous avons conçu la première partie comme l'ensemble des préalables, des bases nécessaires pour comprendre et bien poser le sujet. Nous nous sommes intéressée à la personnalité de l'auteur et avons recensé tous les travaux ayant tenté de retrouver l'identité dudit Dalimil. Comme nous l'avons dit, rien ne nous est parvenu sur ce point et la recherche tchèque s'est très longtemps focalisée sur la résolution de ce mystère. Préférant nous contenter de brosser une proposition de portrait de l'auteur, nous avons refusé de nous prononcer pour une personnalité précise, compte tenu du manque d'informations probantes. Nous nous sommes ensuite consacrée au contexte historique de production : la crise et le paroxysme qu'elle atteint en 1309-1310 (coup d'État de Kutná Hora, rixes à Prague entre les Tchèques et les Misniens) semblent avoir constitué l'impulsion déterminante dans la rédaction de la chronique, et l'histoire joue ensuite un rôle essentiel comme vecteur des idées politiques de l'auteur. Nous avons ensuite 
présenté nos sources primaires, c'est-à-dire les quatorze manuscrits de la chronique aujourd'hui conservés. Nous en avons fait une description codicologique précise, qui s'avéra un outil précieux pour mieux comprendre les utilisations postérieures de ce texte. Pour finir, nous avons présenté les sources convoquées par l'auteur, en essayant d'identifier les œuvres ouvertement mentionnées (ce ne fut pas toujours aisé dans la mesure où celles-ci sont nommées d'après le lieu de conservation de l'époque) et de décrypter les allusions contenues au fil du texte.

La deuxième partie se concentre sur le projet identitaire et l'analyse du message nationaliste contenus dans la Chronique de Dalimil. Nous avons tenu à bien différencier ces deux démarches qui correspondent à deux logiques, deux mouvements bien distincts. La question du projet identitaire vise en effet à pourvoir la nation tchèque d'un ciment fédérateur, à travers la construction d'un passé commun faiseur de sens. Ce premier axe s'articule autour de trois points: 1. la volonté de fonder et de canoniser un genre littéraire proprement tchèque, en rupture avec les héritages latin et allemand dominants, avec une langue spécifique - le vieux-tchèque - et un nouveau système de rimes - les vers hétérométriques -, qui s'imposeront par la suite comme les vers du pamphlet et de la lutte politique ; 2 . l'instrumentalisation du passé et la mise en avant de saints patrons ; 3 . le rôle de l'espace et de la géographie. Le deuxième axe s'interroge sur la question du nationalisme et des étrangers. Nous nous proposons d'interroger l'amalgame qui fait habituellement de la chronique un texte nationaliste et xénophobe. Nous revenons donc sur la notion de "nation ", et essayons de comprendre comment Dalimil la met au jour dans son texte. Si nous insistons sur le ton haineux de ce dernier, nous mettons en avant la concentration de ce texte sur l'antagonisme tchéco-allemand, et affirmons que le message belliqueux appelant à éradiquer l'élément allemand de Bohême ne nous permet pas de conclure pour autant à une xénophobie généralisée à l'encontre de tous les étrangers. En outre, nous entendons proposer une grille de lecture plus fine: nous montrons ainsi que Dalimil est plutôt clément à l'égard de la communauté juive par rapport à ses contemporains, et qu'il témoigne d'une sympathie réelle pour les autres Slaves et les voisins hongrois. Nous insistons par ailleurs sur le rôle du contexte, très spécial en ce début du XIVe siècle, pour comprendre l'attitude de Dalimil envers les Allemands, et signalons l'importance de la colonisation allemande (XIIe siècle) et de la crise généralisée produite par l'interrègne de 1306-1310.

La troisième partie, enfin, porte sur la société de Dalimil et le programme politique qu'il lui adresse. La société occupe une place primordiale dans ce texte, tendu entre une vision noire de la situation contemporaine et une volonté farouche de revenir à un âge d'or perdu. Nous nous efforçons de cerner l'image de la société telle qu'elle transparaît dans ce texte, en pointant d'emblée l'importance du modèle tripartite féodal et des états privilégiés. Seuls comptent en effet la noblesse et le clergé pour Dalimil, tandis que la bourgeoisie - soucieuse de bousculer l'organisation sociale et de proposer un ordre nouveau, en accord avec son importance grandissante - est dépeinte comme le troublefête et comme une force montante extrêmement dangereuse pour l'équilibre sociopolitique. Selon la logique de Dalimil, les nobles sont les seuls à trouver une place dans le projet national qu'il propose : par le rôle politique que leur confère leur statut, ils sont les seuls acteurs responsables, capables de dépasser leur intérêt personnel au profit d'un devoir supérieur. À l'inverse les roturiers (et à leur tête les bourgeois) incarnent les manœuvres les plus sordides que l'homme vil est prêt à accomplir, dans le but de son ascension sociale et de son enrichissement. Surtout, Dalimil s'évertue à préserver le rôle 
prépondérant de la noblesse à un moment où celui-ci est de plus en plus remis en question. C'est là qu'intervient la portée politique de son texte. Nous n'hésitons pas, en effet, à qualifier la Chronique de Dalimil de programme politique. Dans une société qui ne reconnaît pas encore le politique comme un champ bien distinct, l'histoire, l'appropriation d'un passé commun, étaient la matière privilégiée par les auteurs désireux de formuler leurs attentes dans ce domaine. Nous sommes donc attentive à la prise de conscience de ce champ par Dalimil, et aux idées qu'il étaie tout le long de son discours. Brandissant les dangers qu'incarnent les Allemands et le patriciat des villes, il appelle la noblesse à se ressaisir et à circonscrire le pouvoir du souverain, afin de renouer avec le bon gouvernement d'avant la Bulle d'or de Sicile (1212) et les dérives absolutistes qu'elle a matérialisées. Nous mettons en avant le rôle déterminant qu'il accorde à la noblesse et au conseil, comme lieu où s'actualise le prétendu "contrat» qui unit le souverain et ses seigneurs, depuis que Przemysl le Laboureur a été fait duc - d'après le mythe de Libuše qui figure au début de la chronique. L'histoire, l'invention de précédents pour justifier le bien-fondé de l'élection du souverain, ou encore le droit au tyrannicide font office de véritables arguments dans ce texte, pleinement imprégné de l'idée que la preuve de l'ancienneté équivaut à la forme de légitimation la plus valeureuse. Le discours de Dalimil relève clairement du mode performatif et entend produire une réaction précise au sein du public. C'est d'ailleurs ce dernier qui est pris pour objet dans le dernier point de cette partie. À partir des quatorze manuscrits de la chronique, copiés aux XIVe et XVe siècles, nous avons entrepris de saisir les motivations des copies successives à travers l'environnement textuel des différents codices, les ajouts et les mentions marginales. Ce travail nous a permis de situer dans le temps le décalage entre les visées premières de l'auteur - tendues essentiellement vers le double projet de préservation de la souveraineté nationale et de proposition d'un idéal de gouvernement - et une réception de plus en plus restreinte de son œuvre, tendant à limiter la portée de ce texte aux mots nationalistes et germanophobes qu'il contient. L'un des manuscrits médiévaux de la chronique, celui dit de Cerroni (1447), symbolise à lui seul cette évolution très nette et précoce, dans la mesure où il est contenu dans un codex qui renferme exclusivement des textes germanophobes et nationalistes. Très tôt, l'instrumentalisation de ce texte, comme mot d'ordre de combat, s'est donc placée en rupture avec l'idéal de concorde prôné par l'auteur, quand bien même cet idéal était artificiel et revêtait surtout une fonction rhétorique.

Dans le cadre de cette thèse de doctorat, nous avons également proposé une traduction de la Chronique de Dalimil, dans la mesure où notre objectif était de faire connaître cette source d'une grande richesse à un public non tchécophone. Ce texte n'avait jamais été traduit dans aucune langue étrangère moderne jusque-là, les entreprises qui ont précédé remontant toutes au Moyen Âge. Les deux traductions allemandes qui existent datent en effet du XIVe siècle et sont le résultat de la volonté de la communauté allemande de Bohême de se distinguer des Allemands fustigés dans le texte: ces deux versions s'appliquent à construire une image spécifique des Allemands de Bohême, image inexistante dans la version originale dans la mesure où, pour Dalimil, ces derniers n'étaient que des suppôts de ceux qui, campés derrière les frontières, attendaient leur heure pour s'emparer de la Bohême. La traduction latine (vers 1330-1340), retrouvée sous la forme d'un fragment à Paris en 2005, semble quant à elle avoir été motivée par le désir de rendre accessible - à un destinataire non tchécophone - la chronique la plus complète et la plus récente de l'époque sur l'histoire du peuple tchèque. En dehors de son caractère lacunaire, ce texte présente d'importantes imperfections sur le plan linguistique, comme 
des calques du tchèque et des erreurs de traduction malheureuses; la priorité était, semble-t-il, de produire un manuscrit somptueux comme l'indique la richesse des enluminures. Notre traduction est donc la première à tenter de retranscrire, dans toute sa teneur, le texte original tchèque. Nous nous sommes appuyée pour ce faire sur le manuscrit de Vienne qui est, d'après les nombreux travaux des philologues, celui qui se rapproche le plus du manuscrit princeps aujourd'hui perdu. Nous proposons une traduction qui reproduit la forme versifiée, mais avons abandonné les rimes. Nous avons accompagné cette traduction d'une introduction présentant les difficultés auxquelles nous avons dû faire face durant cette tâche. 